\title{
Torsion du cordon spermatique sur testicule non descendu (A propos de deux cas et revue de la littérature)
}

\author{
S. BENNANI, Z. DAHAMI, R. RABII, A. DEBBAGH, A. JOUAL, M. ELMRINI, S. BENJELLOUN. \\ Service d'Urologie, CHU Averroès, Casablanca, Maroc
}

\section{RESUME}

La torsion sur testicule non descendu reste une affection exceptionnelle. Elle survient le plus souvent chez l'adulte jeune ; son étiopathogénie est mal connue.

Deux observations colligées dans le service d'urologie du centre hospitalier Averroès de Casablanca sont rapportées. Le tableau clinique comporte une douleur abdominale d'apparition brutale sans fièvre avec une masse inguinale palpable et une vacuité scrotale homolatérale. L'échographie abdominale met en évidence une masse d'échostructure tissulaire hétérogène. L'exploration chirurgicale faite en urgence retrouve un testicule nécrosé en ectopie inguinale dans les deux cas. Une orchidectomie est réalisée et l'étude anatomopathologique ne montre pas de signe de malignité.

La prise en charge des torsions sur testicules non descendus consiste en une exploration chirurgicale urgente. La place de l'imagerie semble être restreinte. L'abord inguinal permet de réaliser le diagnostic, la détorsion, l'abaissement et la fixation dans la bourse homolatérale en cas de testicule viable.

Mots clés : Testicule non descendu - Torsion du testicule.

\section{INTRODUCTION}

La torsion du cordon spermatique sur testicule non descendu est une urgence chirurgicale rare. Elle provoque par strangulation mécanique du cordon, une interruption brutale de la vascularisation testiculaire mettant en jeu, par ischémie aiguë, la vitalité de ce dernier, imposant ainsi un diagnostic et une intervention rapides pour conserver un testicule viable, et préserver de meilleurs espoirs de fertilité.

\section{OBSERVATION $n^{\circ} 1$}

R.G. âgé de 15 ans présente depuis une semaine des douleurs pelviennes gauches isolées. L'examen clinique retrouve une vacuité scrotale gauche et un testicule droit en place. Les orifices herniaires sont libres.

L'échographie objective (Figure 1) une masse latéro-pelvienne gauche de $7,5 \times 6 \times 4 \mathrm{~cm}$ de diamètre et contenant une formation échogène discrètement hétérogène de 4,6 x 3,1 x $3,5 \mathrm{~cm}$ de diamètre en rapport avec le testicule gauche non descendu.

L'exploration chirurgicale est décidée. La voie d'abord est une incision inguinale gauche. Après contrôle premier du cordon spermatique, où un prélèvement sanguin a été fait pour dosage des marqueurs tumoraux ( $\beta \mathrm{HCG}$

Correspondance : Dr Zakaria Dahami, 113, avenue de Nice, Casablanca Maroc

Tel/fax :022 267231 - E.mail : zdahami@hotmail.com 


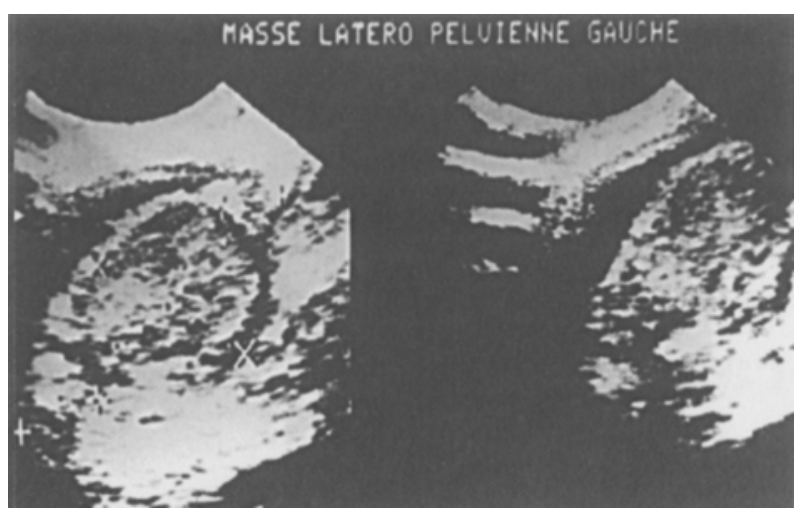

Figure 1: Échographie abdomino-pelvienne : Tesicule non descendu en position inguinale gauche.

et $\alpha \mathrm{FP}$ ), la libération de la masse retrouve un aspect tumoral. Une orchidectomie gauche est réalisée. L'étude histologique de la pièce opératoire met en évidence une nécrose ischémique du parenchyme testiculaire sans signe de malignité.

Le dosage des marqueurs tumoraux est négatif.

\section{OBSERVATION $\mathrm{n}^{\circ} 2$}

O.A. âgé de 19 ans, présente depuis 3 jours, une douleur atroce localisée au niveau de la fosse iliaque gauche avec asthénie, anorexie évoluant dans un contexte apyrétique. L'examen clinique retrouve une masse inguinale gauche mobile, dure et douloureuse à la palpation. L'examen des organes génitaux externes retrouve un testicule droit en place avec une vacuité scrotale gauche. L'échographie met en évidence une masse d'échostructure tissulaire hétérogène bien limitée de forme ovalaire mesurant $3,4 \times 1,3 \times$ $3,4 \mathrm{~cm}$ entourée d'une petite lame d'épanchement liquidien.

L'exploration chirurgicale par voie inguinale découvre une torsion du cordon spermatique gauche sur un testicule en ectopie pelvienne. Ce dernier est de couleur noirâtre et ne reprend pas sa coloration après détorsion et imbibition au sérum tiède. On réalise alors une orchidectomie.

L'étude anatomopathologique retrouve un parenchyme testiculaire en nécrose ischémique sans signe de malignité.

\section{DISCUSSION}

Le premier cas de torsion du cordon spermatique sur testicule non descendu signalé dans la littérature $[4,7,11,21,24,25]$ a été rapporté par GERSTER en 1897, il s'agissait d'un sarcome [in 24]. YANICELLI en 1948 a publié une série de 4 cas [in 1].

JOHNSON et HOLMES ont décrit, en 1964, des cas de torsion sur testicule ectopique chez. des patients souffrant de maladies neuromusculaires spasmodiques [22]. Jusqu'en 1984, 26 cas ont été rapportés chez des sujets ayant des maladies neuromusculaires spasmodiques [26].

La fréquence de cette affection est faible, HAND [8] sur une série de 153 cas de torsion du cordon spermatique, ne retrouve que trois cas de testicules non descendus $(1,9 \%)$. C'est une complication qui survient le plus souvent chez le sujet jeune avec un maximum de fréquence entre 14 et 15 ans $[8,12,19]$.

Si l'étiopathogénie de la torsion du cordon spermatique en position normale est claire, en rapport le plus souvent avec un déficit des moyens de fixation, celle de la torsion du testicule ectopique reste mal connue [20]. Plusieurs mécanismes ont été invoqués dans la genèse de la torsion sur testicule non descendu :

Une contraction spasmodique du muscle crémaster après effort physique $[1,19,22]$.

L'augmentation du volume testiculaire $[6,13]$.

La mobilité du testicule dans la cavité abdominale [13].

Le massage réalisé par les ondes péristaltiques des anses intestinales [13].

La disposition angulaire du cordon spermatique [3].

Les signes fonctionnels sont dominés par la douleur d'apparition brutale, intense siégeant au niveau de la région inguinale ou de la fosse iliaque qui s'atténue après quelques heures témoignant d'un processus de nécrose en cours [23]. Elle constitue le symptôme initial dans $80 \%$ des cas $[8,9]$.

Des troubles digestifs tels des nausées, vomis- 
sements, ténesmes et une diarrhée peuvent accompagner la douleur [2, 4, 24]. La survenue d'une hématurie est exceptionnelle [1].

La palpation de l'abdomen retrouve une douleur qui siège au niveau de la fosse iliaque et de la région hypogastrique, et une masse quand le testicule est en position inguinale [12, 141.

L'examen des organes génitaux externes retrouve une vacuité scrotale homolatérale. Le reflexe crémastérien est absent en cas de torsion du cordon spermatique [29]. Cependant, ce signe peut être retrouvé au cours d'une hernie inguinale étranglée ou d'une tumeur testiculaire [5].

Le toucher rectal peut être douloureux et peut mettre en évidence l'existence d'une masse en rapport avec une tumeur testiculaire $[4,12$, 14].

A ce stade clinique, le diagnostic différentiel se pose essentiellement chez l'enfant avec une appendicite aiguë, une hernie inguinale étranglée, une abcédation ou une tumeur testiculaire.

Chez l'adulte, en plus de ces diagnostics, on peut discuter une orchi-épididymite aiguë ou une colique néphrétique $[2,15,23]$.

L'échographie abdominale n'est pas un examen fiable pour le diagnostic de torsion testiculaire ectopique [23]. Sa sensibilité est de 13 à $17 \%$ lorsque le testicule n'est pas palpable. La torsion se caractérise par un aspect hypoéchogène du testicule au début, pour devenir hétérogène par la suite. Seule la mise en évidence indirecte de la spire de torsion serait pathognomonique [23].

L'échographie Doppler couleur peut confirmer lors d'une torsion l'absence de flux sanguin au niveau du testicule [2, 23].

Ia TDM a une bonne sensibilité si le volume du testicule est supérieur à $1 \mathrm{~cm}$ et lorsque sa localisation est canalaire ou à proximité de l'orifice inguinal profond [10]. L'imagerie par résonance magnétique a une sensibilité de $88 \%$ et une spécificité de $100 \%$ [20].

Ia scintigraphie au pertechnate de sodium marqué au Technétium 99 M a une sensibilité de $95 \%$ et une spécificité de $100 \%$, et montre un testicule hypofixant en cas de torsion [27]. La complexité de cet examen rend cette exploration impossible en pratique d'urgence [23].

Le traitement d'une torsion sur testicule non descendu est une urgence chirurgicale à réaliser dans les six premières heures, il ne doit être retardé en aucun cas par un examen complémentaire.

Ce traitement a pour objectif de rétablir la vascularisation testiculaire par une détorsion en comptant le nombre de spires, de prévenir la récidive en fixant le testicule après son abaissement et de prévenir une torsion controlatérale par une orchidopexie. Le contrôle premier du cordon est impératif si une tumeur du testicule est suspectée. L'orchidectomie est pratiquée lorsque le testicule n'est pas viable. Dans les cas intermédiaires, la décision thérapeutiques peut être difficile à prendre, et prendra en considération, de façon subjective, des arguments de durée d'ischémie, du nombre de spires, et de la rapidité de recoloration du testicule.

Pour certains auteurs, l'orchidopexie controlatérale préventive doit être réalisée dans tous les cas, car le risque de survenue d'une torsion est de 3 à $18 \%[13,17]$.

Le risque de cancérisation d'un testicule non descendu est 35 à 50 fois supérieur à celui d'une glande en place $[7,8,16,18,28$ I. Le type histologique le plus fréquement retrouvé est le séminome (52\% des cas), suivi du sarcome (18\%), du teratome (15\%), du carcinome embryonnaire (10\%) et enfin le choriocarcinome $(5 \%)[14,21,28]$.

Après traitement, l'avenir de la majorité des testicules reste incertain, et plusieurs complications évolutives peuvent survenir : atrophie testiculaire, stérilité ou hypofertilité, fonte purulente du testicule et dégénérescence.

La constatation d'une tumeur du testicule sur la pièce d'orchidectomie fait réviser le pronostic et les mesures thérapeutiques complémentaires en fonction du type histologique et $\mathrm{du}$ bilan d'extension. 


\section{CONCLUSION}

La torsion sur testicule non descendu est une urgence chimurgicale rare qui touche préférentiellement l'adulte jeune, et dont le diagnostic est essentiellement clinique. Son pronostic est conditionné par l'ancienneté de la torsion. Le taux de sauvetage est optimal lorsque la chirurgie a lieu tôt.

\section{REFERENCES}

1. ABU-DALU I., URCA I. : Torsion du testicule ectopique. Helv. Chir. Acta., 1973, $40:$ 413-416.

2. AUBERT D., VALIOULIS I. : Torsion du cordon spermatique. Rev. Prat., 1999, 49 : 327-331.

3. BERUTTI.A, BOUVIER.B. : Torsion du cordon spermatique et des annexes du testicule. Encylop. Méd. Chir., Urol. Gynecol., 1980, 41415, 4-7.

4. BOSCHI L., MANDRIOLI M. : Torsion of a pelvic testicle in an adult. A clinical case. Minerva Chir., 1991, $46: 341-343$.

5. CILENTO B.G., NAJJAR S., ATALA A. : Cryptorchidism and testicular torsion. Pediatr. Clin. North. Am., 1993, 40 : 1133-1149.

6. CHOU H.Y., CHEN C.H., HUANG C.J., LI H.H., HUANG C.H. : Torsion of a malignant undescended testis. Kao Hsiung I Hsueh Ko Hsueh Tsa Chih, 1998, 14 : 308-310.

7. DUNCAN N.D., GABAY L., COARD K.C., ANTOINE M. : Torsion of an intra-abdominal testicale in a neonate. Case report and review of the literature. West. Indian. Med. J., 1997, 46 : 126-127.

8. EIN S.H. : Torsion of an undescended benign tesicular teratoma. J. Pediatr. Surg., 1987, 22 : 799-801.

9. FONKALSRUD E.W. : Testicular undescent and torsion. Pediatr. Clin. North. Am., 1987, 34 : 1305-1317.

10. FONTAINE E., HAJRI M., BEURTON D. : Cryptorchidie. Encylop. Méd. Chir., Néphrol. Urol., 1995, 18-620-A-10.

11. FRANK R.G., GERARD P.S., BARBERA J.T., LINDSAY K. : Torsion of an intra-abdominal testis tumor presenting as anacute abdominal. Urol. Radiol., $1990,12: 50-52$.

12. GARBER H.E., KAUFFER G.I. : Torsion of intraabdominal testicular seminoma. J. Urol., 1968, 98 : 684-685.

13. GERAUD M., LOC'H P., BRON M. : Acute complica- tions of testicular ectopy. Chir. Pediat., 1989, 30 : 145-146.

14. HUTCHESON J.C., KHORASANI R., CAPELOUTO C.C., MOORE F.D., SILVERMAN S.G., LOUGHLIN K.R. : Torsion of intra-abdominal testicular tumors. A case report. Cancer, 1996, $77: 339-343$.

15. MOREL JOURNEL N., VALIGNAT C., LOPEZ J.G., PERRIN P. : Torsion du testicule et de ses annexes. Rev. Prat., 1998, $48:$ 2119-2122.

16. LEISSNER J., FILIPAS D., WOLF H.K., FISCH M. : The Undescended testis : considerations and impact on fertility. Br. J. Urol., 1999, $83: 885-892$.

17. LUGAGN E., DELPON P.M. : Cancer du testicule. Rev. Prat., 1999, 49 : 216-222.

18. MOURIQUAND P. : Testicules non descendus. Rev. Prat., 1998, 48 : 2110-2114.

19. MOWAD J.J., KONVOLINKA C.W. : Torsion of undescended testis. Urology, 1978, $12: 567-568$.

20. NESA S., LORGE F., WESE F.X., NJINOU B., OPSOMER R.J., VAN CANGH P.J. : Testicular torsion after previous orchydopexy for undescended testis. Acta Urol. Belg., 1998, 66 : 25-26.

21. PESSI T.T. : Torsion of intra-abdominal testicle. A case report. Ann. Chir. Gyn., 1968, 57 : 557-559.

22. PHILIPPS N., HOI.MES W.T. : Torsion infraction in ectopic cryptorchidism : a rare entity occuring most commonly with spastic neuromuscular disease. Surgery, 1972, 71 : 335-338.

23. RAMBEAUD J.J., GREATOREX R.A. : Torsion du testicule et de ses annexes. Encylop. Méd. Chir., Néphrol. Urol., 1991, 18622-A-10.

24. RASKOV H., BOESGAARD S. : Torsion of intra-abdominal testicle. Case report. Acta. Chir. Scand., 1989, $155:$ 483-484.

25. RIEGLER H.G. : Torsion of intra-abdominal testis. An unusual problem in diagnosis of the acute surgical abdomen. Surg. Clin. North. Am., 1973, 52 : 371 374.

26. SCHULTZ K.E., WALKER J. : Testicular torsion in undescended testis. Ann. Emerg. Med., 1984, 13 : 567-569.

27. SCHMITT M. : Torsion du cordon spermatique. Rev. Prat., 1995, 45 : 1687-1689.

28. SRIKUMAR B., PII AI M.D. : Pediatric testicular problems. Pediat. Clin. North. Am., 1998, 45 : 813-830.

29. TOZAWA K., WASHIDA H., HONMA H., YAMADA Y.: Torsion of undescended testis detected with testicular imaging. Hinyokika Kiyo, 1993, 39 : 377-379. 


\section{ABSTRACT \\ Torsion of spermatic cord of undescended testis}

S. BENNANI, Z. DAHAMI, R. RABII, A. DEB-

BAGH, A. JOUAL, M. ELMRINI, S. BENJEL-

LOUN.

Torsion of an ectopic testis is an exceptional disease and mostly occurs in young adults. Its etiopathogenesis has not been elucidated.

The authors report two cases. The clinical presentation consisted of sudden onset of abdominal pain without fever, associated with a palpable inguinal mass and a homolateral empty scrotum. Abdominal ultrasound showed a mass with a heterogeneous echogenicity. Emergency surgical exploration revealed a necrotic inguinal ectopic testis. Orchidectomy was performed and pathological examination did not reveal any signs of malignancy.

The management of torsion of an undescended testis revealed by a mass with homolateral empty scrotum consists of urgent surgical exploration.

Medical imaging appears to have a limited role. An inguinal incision allows diagnosis, detorsion, reduction and fixation in the homolateral scrotum.

Key-Words: Undescended testis - Torsion. 